Meta

Journal des traducteurs

Translators' Journal

\title{
La traduction de la Bible en boulou
}

\section{Bilounga Belinga Sindeu}

Volume 22, numéro 3, septembre 1977

URI : https://id.erudit.org/iderudit/004159ar

DOI : https://doi.org/10.7202/004159ar

Aller au sommaire du numéro

Éditeur(s)

Les Presses de l'Université de Montréal

ISSN

0026-0452 (imprimé)

1492-1421 (numérique)

Découvrir la revue

Citer cet article

Sindeu, B. B. (1977). La traduction de la Bible en boulou. Meta, 22(3), 192-194.

https://doi.org/10.7202/004159ar d'utilisation que vous pouvez consulter en ligne.

https://apropos.erudit.org/fr/usagers/politique-dutilisation/ 


\section{La traduction de la Bible en boulou}

Georges Mounin a écrit que «pour traduire une langue étrangère, il faut remplir deux conditions dont chacune est nécessaire et dont aucune en soi n'est suffisante : étudier la langue étrangère ; étudier (systématiquement) l'ethnologie de la communauté dont cette langue est l'expression». Si ce jugement de Mounin se justifie pour la langue de départ, il est également valable pour une langue d'arrivée. En effet, les différences écologiques et culturelles posent au traducteur des problèmes énormes, auxquels il ne trouve pas toujours de solution.

Le traducteur a pour mission de transmettre un message qui puisse, entre autres, provoquer chez ses auditeurs les mêmes réactions que chez les auditeurs de la langue de départ. Si l'on tient compte du fait que les langues n'ont pas les mêmes structures lexicales, ni la même syntaxe, et que les mots n'ont pas la même signification pour des individus différents et dans des civilisations différentes, on peut supposer que le traducteur doit parfois faire un gros effort pour trouver, quand cela est possible, des équivalences entre la langue de départ et la langue d'arrivée.

Dans le cas qui nous intéresse, la traduction de la Bible en boulou, nous savons que les civilisations hébraïque et boulou diffèrent à tous les points de vue, ou presque. Le traducteur de la Bible a donc été confronté à des problèmes beaucoup plus graves que s'il avait eu à traduire du français vers l'anglais, par exemple. Ces problèmes sont d'abord d'ordre écologique.

Les Boulou vivent dans le sud du Cameroun, en pleine forêt équatoriale. Or, l'Ancien Testament fait souvent mention du désert. Ce mot a été traduit en boulou par nkôt si, c'est-à-dire terre sans eau. Il est très difficile au fidèle boulou d'imaginer une région dépourvue de rivières et de sources, et il lui est encore plus difficile de se représenter les vastes étendues sablonneuses du désert, lui qui est presque étouffé par l'épaisseur de la forêt.

Les noms des arbres ont tout simplement été laissés en français, car comment traduire olivier, sycomore, vigne... dans une langue où ces arbres ne sont pas nommés? Il en est de même pour les animaux. Mais ici, le traducteur a parfois eu recours à des substituts. Si le mot chameau est cité en français dans le texte boulou, loup a été traduit par ze qui signifie panthère, animal dans la 
civilisation boulou qui a à peu près la même valeur symbolique que le loup chez les Européens.

Comme le souligne avec raison Nida, « il y a des difficultés nées du fait que les «choses » à traduire dans une langue n'existent pas dans la culture correspondante à cette langue et ne s'y trouvent pas nommées s.

Les instruments de musique ne sont pas les mêmes chez les Hébreux et chez les Boulou. À la place de cor, flûte, cymbale..., le traducteur a substitué tam-tam, tambour, balafon... Le blé, l'avoine et l'orge ont été traduits par bidi c'est-à-dire produits vivriers.

Certains mots ont été traduits par leur définition. Ainsi tentes devient menda bindélé, ce qui veut dire les maisons faites avec de la toile.

Scribes est rendu par betili bekalate c'est-à-dire ceux qui écrivent les livres ou les lettres, car il n'y a qu'un mot pour désigner livre et lettre, seul le contexte indique de quoi il est question, ce qui n'est pas le cas pour la traduction de scribes. Le mot hôtellerie a été plus difficile à traduire. Il devient en boulou nda beyen qui veut dire maison des étrangers. Ceci ne signifie absolument rien, car ici les étrangers dorment toujours dans la même maison que leurs hôtes; de plus, l'idée de logement contre paiement n'est pas traduite. Encore une fois, le traducteur n'a pas pu trouver d'équivalent parce que dans la civilisation boulou, on a le devoir d'accorder l'hospitalité aux étrangers.

Rendez à César ce qui est à César devient va'ane kéza biôm bi kéza c'està-dire donnez au roi ce qui est au roi, ce qui signifie exactement la même chose dans les deux contextes.

Certains passages, par contre, ont été très mal traduits faute de termes correspondants et surtout à cause d'une connaissance insuffisante de la langue d'arrivée.

Les moutons n'ont pas de laine au sud du Cameroun. Pour traduire laine, le traducteur a choisi mevul, mot qui n'est utilisé chez les Boulou que pour désigner les poils du pubis de la femme. Ceci provoque toujours des réactions parmi les fidèles.

Dans le pays boulou, il n'y a rien dont la blancheur soit comparable à celle de la neige. Plus blanc que la neige a été traduit par éfumulu ane neige. Or, ce mot barbare pour les Boulou (et qui pose des difficultés de prononciation aux vieux catéchistes) n'évoque pas chez les fidèles le degré de blancheur qu'il représente. Si le traducteur avait eu une meilleure connaissance de la langue, il se serait aperçu qu'il y a en boulou une expression équivalente, qui est éfumulu ne fuum c'est-à-dire très très blanc, d'un blanc pur, sans tache.

Dans I Samuel $18: 25$, Saül demande à David de ramener des prépuces de Philistins. Or le boulou n'a pas de terme pour désigner le prépuce, mais il distingue par des noms différents un membre viril circoncis et un membre viril non circoncis. Prépuce a été traduit par le mot qui désigne en boulou les membres virils non circoncis; cela fait croire aux fidèles que Saül a demandé à 
David de ramener les membres virils des Philistins, ce qui est pour eux le comble de la cruauté.

Parfois, certaines précisions n'ajoutent rien au texte boulou. Quand on parle du sel qui a perdu sa salinité, dire qu'il n'est plus bon qu'à être foulé aux pieds n'ajoute rien quant à l'effet que l'idée de jeter son sel (denrée précieuse pour le Boulou) produit sur une ménagère boulou.

Malgré l'absence de termes correspondants, certaines expressions ont été très bien rendues. Vu que la profession du berger n'existe pas dans la civilisation en question, le Seigneur est mon berger devient Jéhovah ane mba'ale wom ce qui signifie Jéhovah est mon gardien.

Le langage ne couvre pas tous les aspects d'une civilisation mais, comme dit Meillet : "Tout vocabulaire exprime une civilisation.» Ceci nous amène à conclure avec Mounin que la traduction n'est pas toujours possible et qu'elle ne l'est que dans une certaine mesure, surtout quand les civilisations concernées sont très différentes l'une de l'autre.

Il est plus aisé de traduire vers sa langue maternelle, car on saisit mieux toutes les subtilités linguistiques, et on trouve plus facilement les équivalences. Or, les missionnaires ont appris les langues indigènes durant une période relativement courte, ce qui ne leur a pas permis de connaître ces langues à fond.

Cependant, toutes les civilisations ont la notion de la joie, du bonheur, de l'amour, du bien, du mal, de la famille... C'est pourquoi, malgré les grandes différences dans les lexiques, les us, les coutumes et les climats, le message de la Bible a pu être transmis à tous les hommes. La Bible a provoqué, à quelques exceptions près, les mêmes réactions chez tous les croyants du monde, ce qui montre que les traducteurs ont atteint leur objectif, malgré certaines maladresses et le caractère insurmontable de certaines difficultés.

Bilounga Belinga Sindeu 